\title{
Diversité floristique et ethnobotanique des plantes sauvages comestibles dans le Département de Bondoukou (Nord- Est de la Côte d'Ivoire)
}

\author{
Noufou Doudjo Ouattara1, 2, 4, Elodie Gaille3, Fred W. Stauffer 4 \& Adama Bakayoko1, 2 \\ 1. UFR des Sciences de la Nature (SN), Université Nangui Abrogoua, 02 BP 801 Abidjan 02, Côte d'Ivoire. \\ 2. Centre Suisse de Recherches Scientifiques en Côte d'Ivoire, 01 B.P. 1303 Abidjan 01, Côte d'Ivoire. \\ 3. Jardin botanique de Neuchâtel, Chemin du Pertuis-du-Sault 58, 2000 Neuchâtel, Suisse \\ 4. Conservatoire et Jardin Botaniques de la Ville de Genève, Laboratoire de systématique végétale et biodiversité, \\ Chemin de l'Impératrice1, 1292 Chambésy-Genève, Suisse. \\ Auteur pour les correspondances : dojjoo@yahoo.fr
}

Original submitted in on $18^{\text {th }}$ December 2015. Published online at www.m.elewa.org on 29th February 2016 http://dx.doi.org/10.4314/jab.v98i1.5

\section{RÉSUMÉ}

Objectifs: Inventorier et évaluer le niveau de connaissance et d'exploitation des plantes sauvages comestibles dans le Département de Bondoukou au Nord-Est de la Côte d'Ivoire.

Méthodologie et Résultats : Des groupes de discussion (focus group) et des entretiens structurés ont été employés pour recueillir les informations auprès de 4 peuples autochtones dans 7 villages. Les espèces ont été récoltées puis déterminées à l'herbier du CSRS. Au total 81 espèces appartenant à 76 genres et 41 familles sont consommées. Les Malvaceae, les Annonaceae, les Arecaceae (palmiers) et les Fabaceae sont les familles dominantes. Les espèces bien connues et bien consommées représentent $27,14 \%$ des taxons ayant fait l'objet d'une enquête sur le niveau de connaissance et d'exploitation. Les organes les plus consommés sont en ordre décroissant d'importance les fruits $(57,14 \%)$, les feuilles $(26,19 \%)$ et les graines $(10,71 \%)$. Les autres organes consommés sont les écorces $(2,38 \%)$, les fleurs, les sèves et les tubercules avec chacun 1,37\%. L'usage alimentaire de Coelocaryon oxycarpum Stapf (Myristicaceae), espèce endémique d'Afrique de l'Ouest, est révélé pour la première fois.

Conclusion et applications des résultats: Plusieurs espèces recensées dans la zone d'étude ont une importance économique pour les femmes et les enfants. Ces espèces font l'objet d'un commerce local qui apporte des revenus supplémentaires à ces populations. La valorisation (préparation de jus ou extraction d'huile) de certains fruits pourrait apporter une valeur ajoutée à ces espèces. D'autres espèces comme Carapa procera DC (Meliaceae) dont l'usage alimentaire est de moins en moins fréquent pourraient être, cependant, prometteuses en cosmétique. Coelocaryon oxycarpum est utilisée comme épice dans les repas (sauces ou soupes) des femmes qui viennent d'accoucher. Une analyse phytochimique des fruits de cette espèce est à envisager.

Mots-clés : Floristique, ethnobotanique, plantes sauvages comestibles, Bondoukou, Côte d'Ivoire 


\section{ABSTRACT}

Floristic diversity and ethnobotany of edible wild plants in the Department of Bondoukou (North-Eastern Côte d'Ivoire)

Objectives: To inventory and assess the level of local knowledge and use of edible wild plants in the Department of Bondoukou in the north-eastern Côte d'Ivoire.

Methodology and Results: focus groups and structured interviews were used to gather information from four indigenous ethnic groups in 7 villages. Species were collected then identified in the laboratory. In total 81 species belonging to 76 genera and 41 families are consumed. From them the families Malvaceae, Annonaceae, Arecaceae and Fabaceae showed up to be the most intensively used. The well-known and most frequently consumed species represent $27.14 \%$ of the taxa. The most frequently consumed organs in decreasing order of importance are fruits $(57.14 \%$ ) leaves $(26.19 \%)$ and seeds $(10.71 \%)$. The other consumed organs are bark (2.38\%), flowers, saps and tubers with $1.37 \%$ each. The consumption of Coelocaryon oxycarpum Stapf (Myristicaceae), an endemic species of West Africa is reported for the first time.

Conclusion and application of findings: Many species identified in the frame of our study area display a large economic importance among women and children. These species are the subject of a local trade that provides extra income to these populations. The valorization (juice preparation or oil extraction) of certain fruits could bring an added value to these species. Other species to a lesser degree used as food may be, however, promising in cosmetics. Coelocaryon oxycarpum is used as a spice in the meal (soup) of women who have just given birth. A phytochemical analysis of the fruits of this species is required to better assess its importance.

Key-words: Floristic, Botany, ethnobotany, edible wild plants, Bondoukou, Côte d'Ivoire

\section{INTRODUCTION}

La part des plantes sauvages comestibles dans l'alimentation et même la réduction de la pauvreté des populations africaines est très importante (Schreckenberg et al., 2006 ; Iranbakhsh et al., 2009). Plusieurs études ont montré cette importance dans différents pays et à différents niveaux. En Ouganda, il a été prouvé que des plantes sauvages comestibles, telles que Carissa spinarum L. et Physalis minima L., pouvaient considérablement complémenter les plantes exotiques cultivées pour réduire la déficience en vitamine A chez les populations (Musinguzi et al., 2007). Les études de Lamien-Meda et al., (2008) au Burkina Faso ont revelé que de nombreuses plantes sauvages comestibles possèdent un niveau élevé d'activités antioxydantes. Dans les pays sahéliens comme le Burkina Faso et le Sénégal, les plantes sauvages comestibles servent d'aliment de soudure (Ayessou et al., 2008, Thiombiano et al., 2010). En Côte d'Ivoire, les plantes sauvages comestibles n'ont pas encore été inventoriées au plan national contrairement à certains pays comme la Tanzanie (Rufo et al.,
2002) où plus de deux cent cinquante (250) espèces ont été inventoriées parmi lesquelles on peut citer Balanites aegyptiaca (L.) Delile, Hyphaene compressa H. Wendl., Hyphaene petersiana Klotzsch ex Mart., Phoenix reclinata Jacq., Raphia farinifera (Gaertn.) Hyl. et Strychnos spinosa Lam.Cependant quelques études locales ou régionales ont été conduites dans certaines parties du pays (Gautier-Beguin, 1992; Ambé, 2001 ; Kouamé \& Gnahoua, 2008). Ces études ont elles aussi montré le rôle essentiel des plantes sauvages comestibles dans l'alimentation et dans l'amélioration des revenus de nombreuses personnes (Gautier-Beguin, 1992 ; Herzog, 1992). Au niveau nutritionnel, les études biochimiques de Herzog (1992) et Herzog et al., (1994) ont révélé la richesse en nutriments et l'importance dans l'équilibre nutritionnel des populations, de plusieurs espèces dont Aframomum alboviolaceum (Ridl.) K. Scum., Spondias mombin L. et Annona senegalensis Pers. Dans les milieux ruraux de la région du V-Baoulé en Côte d'Ivoire, ces auteurs ont remarqué que le mode d'alimentation des 
populations prédispose les enfants à diverses carences nutritionnelles. Cependant, le constat fait par les nutritionnistes est que ces carences sont rarement observées chez les enfants de la région. Pour Gauthier-Beguin (1992) cela s'explique par la consommation des fruits sauvages comestibles par ces enfants. En d'autres termes, l'apport de ces plantes est d'une nécessité absolue pour l'équilibre nutritionnelle des enfants dans cette zone mais probablement dans tous les milieux ruraux de la Côte d'Ivoire. Cependant, toutes les études menées en Côte d'Ivoire ont révélé la menace de disparition de plusieurs espèces dans le pays mais aussi la perte rapide des savoirs traditionnels liés à l'utilisation de ces plantes. En effet, dans la plupart des régions où ces plantes ont été étudiées, il existe un problème de conservation des ressources végétales. A part un nombre très limité d'espèces, les plantes sauvages comestibles ne sont pas conservées lors des défrichements des champs. Ces plantes sont donc de plus en plus menacées dans leurs habitats naturels du fait de la rareté des terres, consécutive à une augmentation exponentielle de la population et au raccourcissement de l'âge des jachères. Malgré leur importance et les menaces, les politiques publiques visant la valorisation et la conservation/protection des plantes sauvages comestibles sont inexistantes. Ainsi, la domestication de ces plantes n'est pas encore

\section{MATÉRIEL ET MÉTHODES}

Sites d'étude: L'étude a été conduite dans le Département de Bondoukou dans la Région du Gontougo, au Nord-Est de la Côte d'lvoire, de novembre 2013 à juin 2015. Dans le cadre de nos recherches, huit (8) missions sur le terrain ont été effectuées dans la région concernée. La zone d'étude est située dans le secteur sub-soudanais (domaine soudanais), à la limite du secteur mésophile (secteur préforestier ou savanes guinéennes) du domaine guinéen (Guillaumet \& Adjanohoun, 1971) ; entre $8^{\circ} 2^{\prime}$ $36.9018^{\prime \prime} \mathrm{N}$ et $08^{\circ} 17^{\prime} 23.6004^{\prime \prime} \mathrm{N}$ de latitude et entre $3^{\circ}$ $2^{\prime} 22.5558^{\prime \prime} \mathrm{W}$ et $2^{\circ} 31^{\prime} 45.3246^{\prime \prime} \mathrm{W}$ de longitude. Elle se caractérise par des transitions forêts-savanes. Aujourd'hui on note une nette dominance des formations de savanes. Le climat est de type subsoudanais, c'est-à-dire faisant la transition entre un courante dans le pays (Kouamé et al., 2014). Afin de mettre en place un programme performant pour la valorisation de ces importantes ressources, la connaissance de leur diversité, abondance et menaces selon les différentes régions du pays s'impose. Surtout dans le Nord-Est de la Côte d'Ivoire où de telles études n'avaient pas encore été conduites. D'une manière générale, cette partie du pays est l'une des moins connue du point de vue floristique (Chatelain et al., 2011). Pourtant elle représente également l'une des régions à fort taux de déforestation (Barima et al., 2009). L'urgence des études dans cette région se pose également par rapport à l'objectif de préservation du parc national et réserve de biosphère de la Comoé. En effet, la connaissance de la flore et son utilisation par I'homme, dans le Nord-Est de la Côte d'Ivoire, est une condition sine qua non pour une gestion durable de ce parc. La présente étude a été menée dans le but de recenser les plantes sauvages comestibles de cette région afin d'en dégager les problèmes de conservation et les possibilités de valorisation de ces ressources. Pour ce faire, l'étude met en évidence le niveau de connaissance et d'exploitation de ces ressources par les populations. Ces données sont importantes dans le cadre de la mise en place d'un programme de vulgarisation car elles guident dans le choix des espèces dans un tel programme (Ambé, 2001).

climat soudanais du type tropical et le régime tropical humide du type baouléen. Les durées de saisons (sèches, pluvieuses) sont très irrégulières (Morel, 2004). La zone baigne tantôt dans un régime à deux saisons, tantôt dans le régime à quatre saisons (Ouattara, 2001). L'étude a été menée auprès des populations autochtones Abron, Dègha, Koulango et Nafana dans 7 villages: Kiendi Walogo, Kouassi N'Dawa, Laoudi Ba, Laoudi Gan, San-Poro (Borokponko), Sorobango et Zagala. Des enquêtes ont également été réalisées dans le grand marché de la ville de Bondoukou.

Enquêtes ethnobotaniques et recensement des espèces : La première étape de l'étude a consisté à recenser toutes les espèces sauvages comestibles. Des groupes de discussion (focus groups) ont été 
organisés dans tous les villages ciblés. A l'auditoire était posée les questions suivantes : quelles sont les plantes sauvages comestibles que vous connaissez dans votre village ou dans la région? Quels sont les noms locaux utilisés pour nommer les différentes espèces?

Après ces entretiens, une incursion est menée dans la brousse et dans les champs en compagnie de certains villageois qui ont une connaissance avérée des plantes afin de récolter toutes les espèces citées. Des échantillons d'herbiers sont alors réalisés, les organes consommés prélevés et des photographies réalisées. Les plantes ont été ensuite déterminées à l'herbier du Centre Suisse de Recherches Scientifique en Côte d'Ivoire, en adoptant la nomenclature selon la classification phylogénétique (APG III, 2009).

La deuxième phase de l'étude est l'évaluation du niveau de connaissance et d'exploitation des espèces sauvages comestibles. Cette phase a concerné 70 espèces sur les 81 inventoriées. Pour ce faire 105 personnes, âgées de 7 à 79 ans, appartenant aux deux sexes, ont été sélectionnées en raison de 15 personnes par village. Chaque personne a été soumise à une interview structurée composée des questions suivantes :

Pour l'évaluation du niveau de connaissance

\section{RÉSULTATS}

Diversité botanique: Quatre-vingt-une (81) espèces ont été recensées au cours de cette étude. Elles appartiennent à 76 genres et 41 familles. La Figure 1 montre le spectre de répartition des familles. Les familles les plus représentées sont celles des Malvaceae avec $7,41 \%$ des espèces, suivies par les Annonaceae, les Fabaceae et les palmiers (Arecaceae) avec chacune 5 espèces soit $6,17 \%$. A la suite de ces quatre familles viennent les Phyllantaceae et les Rubiaceae avec chacune 4 espèces soit $4,94 \%$ des taxons. Ces six familles, soit $7,56 \%$ des familles, renferment $35,8 \%$ des espèces. Les taxons africains représentent $72,84 \%$ de l'ensemble. La liste des espèces est fournie dans le Tableau 1. Parmi ces espèces seulement deux sont endémiques d'Afrique de l'Ouest. II s'agit de Coelocaryon oxycarpum Stapf (Figure 2) et Cola caricaefolia (G. Don) K. Schum. Les arbres et arbustes sont les formes les plus représentées.

Niveau de connaissance et d'exploitation des espèces: Les niveaux de connaissance et d'exploitation sont présentés simultanément dans le
1. Connaissez-vous cette plante/ce fruit ? (a. oui ; b. non)

Le niveau de connaissance est déterminé en calculant le rapport du nombre de personnes reconnaissant l'espèce sur le nombre de personnes interrogées (ici 105).

Pour l'évaluation du niveau de consommation (ou d'exploitation)

2. Si oui (c'est-à-dire vous la connaissez) l'avez-vous déjà consommé, au moins une fois ? (a. oui ; b. non)

Le niveau d'exploitation ou de consommation d'une espèce est déterminé en calculant le rapport du nombre de personnes ayant consommé l'espèce au moins une fois sur le nombre de personnes reconnaissant l'espèce. Les rapports sont ramenés en pourcentage et les espèces sont alors réparties selon les classes suivantes (Ambé, 2001, Kouamé et al., 2008) :

-une espèce est dite bien connue (ou bien exploitée) si le niveau de connaissance (ou d'exploitation) est compris entre $50 \%$ et $100 \%$

-une espèce est dite moyennement connue (ou moyennement exploitée) si le niveau de connaissance (ou d'exploitation) est compris entre $25 \%$ et $50 \%$ - une espèce est dite peu connue (ou peu exploitée) si le niveau de connaissance (ou d'exploitation) est compris entre $00 \%$ et $25 \%$.

Tableau 2. Dix-neuf (19) espèces (soit 27,14\%) sont bien connues et bien exploitées par les populations. Les espèces les plus connues sont Annona senegalensis, Borassus aethiopum, Carica papaya, Elaeis guineensis (palmier à huile), Parkia biglobosa (néré), Saba senegalensis, Tamarindus indica (tamarinier) et Vitellaria paradoxa (karité). Ces espèces ont été reconnues par toutes les personnes interrogées.

Organes consommés: Les organes prélevés sur les plantes pour la consommation sont les feuilles, les fleurs, les fruits, les écorces, les sèves, les tubercules et les graines. Les fruits sont les organes les plus consommés avec plus de $50 \%$ d'utilisation (Figure 3). La consommation des fleurs a été observée chez une seule espèce. II s'agit de Bombax costatum dont les pétales séchés servent à préparer des sauces. C'est également le cas pour la consommation de la sève, observée seulement chez Raphia hookeri et de la consommation de tubercules, observée uniquement chez Dioscorea dumetorum. 
Mode de préparation ou de consommation: Les organes prélevés sont consommés de plusieurs manières. Cela explique aussi différentes formes ou modes de préparation. Les feuilles sont presque toutes utilisées dans la préparation de sauces à l'exception de Lippia multiflora dont les feuilles servent à la préparation d'une tisane. Toutes ces feuilles sont donc cuites avant leur consommation. En ce qui concerne les fruits et les graines, certains peuvent être consommés directement sans aucune forme de traitement. C'est le cas d'Annona senegalensis (Figure 4A), Diospyros mespiliformis, Flacourtia vogelii, Landolphia heudelotii, Oncoba spinosa, Parinari curatellifolia (Figure 4B), Saba spp, Strychnos spinosa, Vitex spp, Ximenia americana, etc. D'autres subissent des traitements comme le séchage (Figure $4 \mathrm{C}$ ) ou la cuisson. Chez certaines espèces le mucilage ou la pulpe autour des graines peut être consommé directement alors que la graine elle-même subit des transformations pour fournir d'autres produits. On peut citer Adansonia digitata, dont la pulpe blanche farineuse est consommée crue, simple ou mélangée à de l'eau et les graines (Figure 4D) sont torréfiées puis écrasées pour donner un condiment utilisé dans la préparation des sauces. Parkia biglobosa et Vitellaria paradoxa (Figure 4) rentrent également dans cette catégorie de plantes. Tout comme Vitellaria paradoxa, les graines de Pentadesma butyracea (Figure 4F) servent à préparer un beurre (ou huile) utilisé dans la cuisine. En ce qui concerne le palmier Borassus aethiopum (rônier), c'est le fruit qui est cuit pour en extraire le jus (Figure 5). Les écorces sont utilisées chez deux espèces (Pouteria alnifolia et Grewia carpinifolia) où elles servent à la fermentation alcoolique de boissons locales.

Valeur socio-économique des espèces: Certaines espèces font l'objet d'un commerce local tenu par les femmes. Parmi ces espèces on distingue celles dont les fruits ou les graines sont vendus dans les marchés locaux en entier: Ricinodendron heudelotii, Saba senegalensis, Saba comorensis, Xylopia aethiopica, Coelocaryon oxycarpum, Monodora myristica, Landolphia heudelotii et Tamarindus indica. D'autres espèces, par contre, sont transformées pour obtenir des huiles (beurre) ou des condiments qui sont vendus: Vitellaria paradoxa, Parkia biglobosa, Adansonia digitata. Les espèces Pentadesma butyracea, Carapa procera et Lophira lanceolata fournissent des huiles mais celles-ci sont rarement vendues sur les marchés. La cueillette et la vente de Saba senegalensis, Saba comorensis et Vitex doniana sont le plus souvent exercées par les enfants. Coelocaryon oxycarpum a une forte valeur socioculturelle dans la région. En effet, les fruits de la plante, qui arrivent à maturité pendant le mois de mars et disponibles jusqu'à la fin du mois de juin, sont ramassés et séchés au soleil par les femmes, pendant cette période. Les fruits séchés sont alors conservés soit pour un usage domestique soit pour être vendus. Ces fruits constituent une épice bien prisée par les personnes qui la connaissent. Ils sont utilisés préférentiellement pour la préparation des sauces (soupes) des femmes qui viennent d'accoucher. 


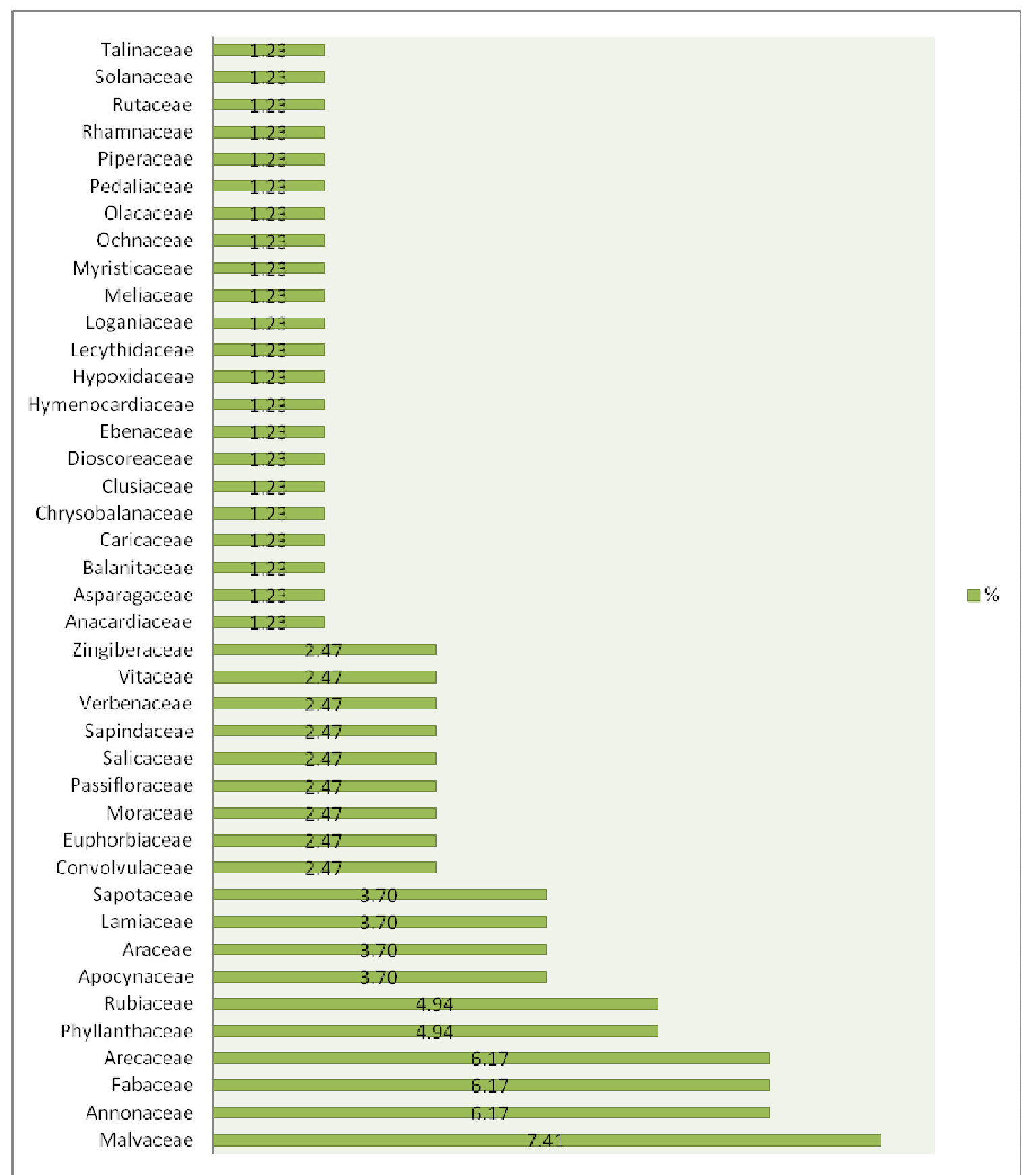

Figure 1: Spectre de répartition des espèces sauvages comestibles dans les familles botaniques de la classification phylogénétique (APG III) 
Ouattara et al. J. Appl. Biosci. 2016 Diversité floristique et ethnobotanique des plantes sauvages comestibles dans le Département de Bondoukou (Nord- Est de la Côte d'Ivoire)

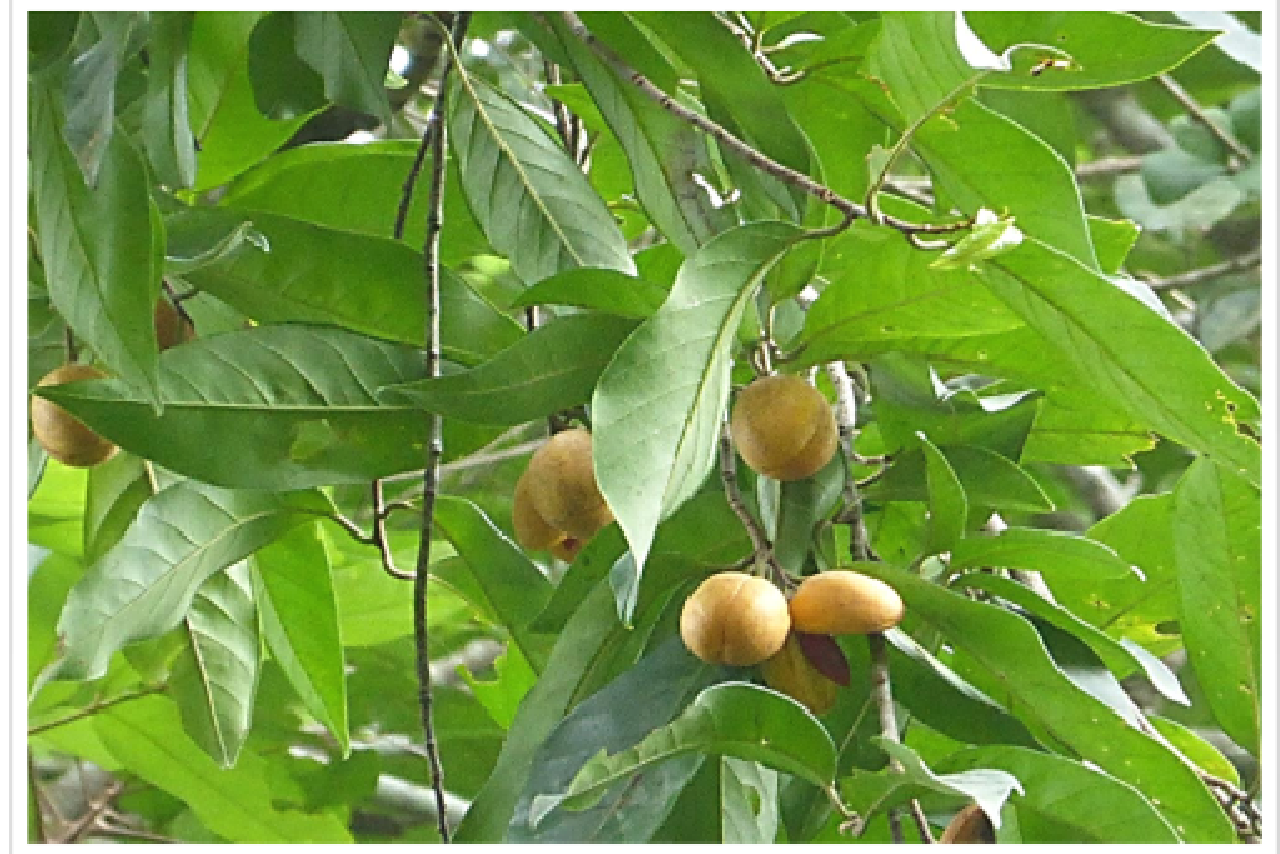

Figure 2: Organes végétatifs et reproductifs de Coelocaryon oxycarpum Stapf., espèce endémique d'Afrique de l'Ouest

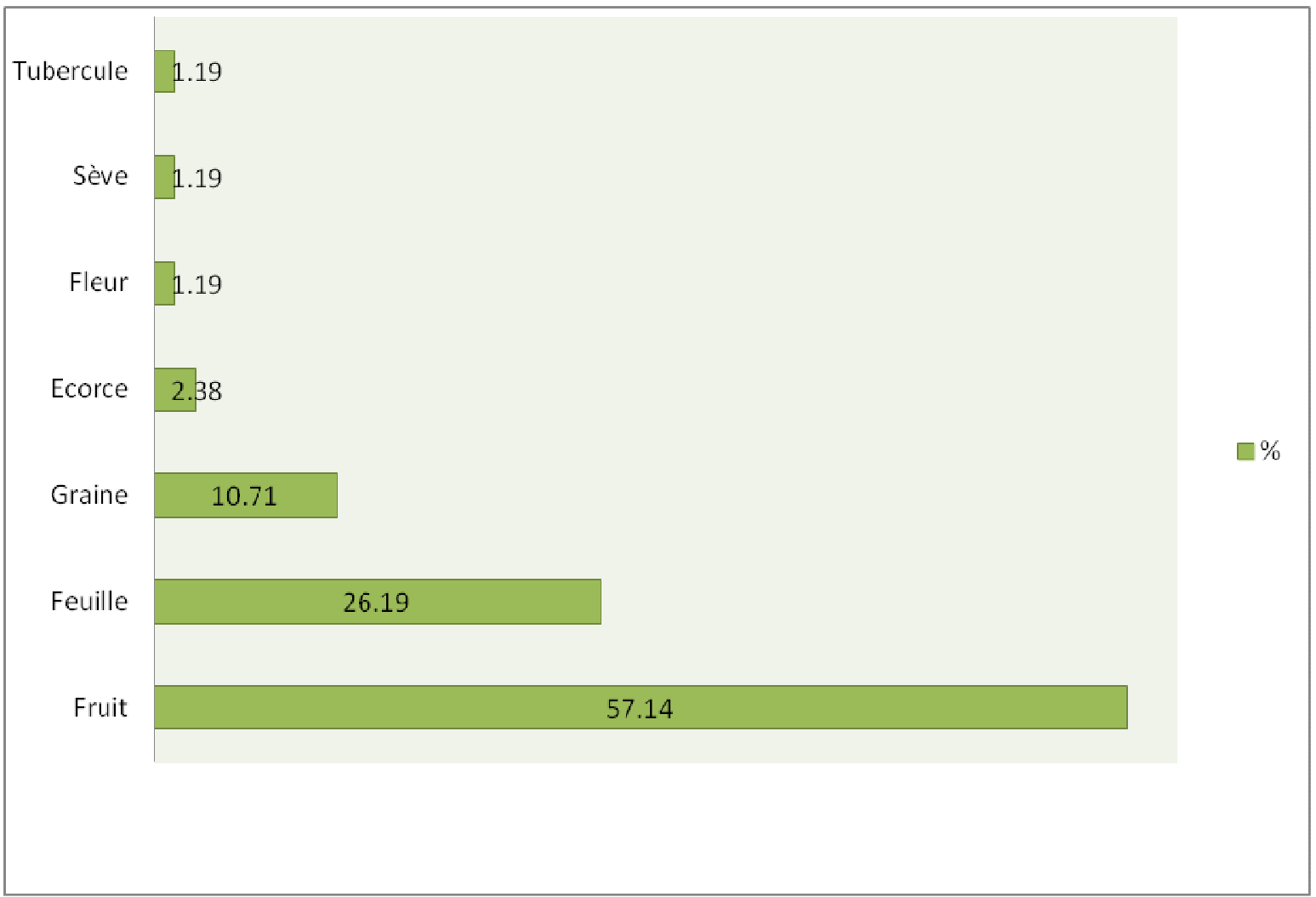

Figure 3 : Proportion d'utilisation des organes prélevés sur les plantes 


\section{Ouattara et al. J. Appl. Biosci. 2016 Diversité floristique et ethnobotanique des plantes sauvages comestibles dans le Département de Bondoukou (Nord- Est de la Côte d'Ivoire)}

Tableau 1. Diversité botanique et chorologie des espèces sauvages comestibles recensées dans le département de Bondoukou

\begin{tabular}{|c|c|c|c|}
\hline Espèces & Famille & Morphologie & $\begin{array}{c}\text { Chorologie et type } \\
\text { biologique }\end{array}$ \\
\hline $\begin{array}{l}\text { Adansonia digitata L. } \\
\text { Adenia cissampeloides (Planch. ex Hook.) } \\
\text { Harms } \\
\text { Aframomum alboviolaceum (Ridl.) K. Scum. } \\
\text { Aframomum melegueta K. Schum. } \\
\text { Afzelia africana Sm } \\
\text { Anchomanes welwitschii Rendle } \\
\text { Annona senegalensis Pers. } \\
\text { Antidesma venosum Tul. } \\
\text { Balanites aegyptiaca (L.) Delile } \\
\text { Blighia sapida K.D. Koenig } \\
\text { Bombax costatum Pellegr.\& Vuillet } \\
\text { Borassus aethiopum Mart. } \\
\text { Bridelia ferruginea Benth. } \\
\text { Carapa procera DC } \\
\text { Carica papaya L. } \\
\text { Cayratia gracilis (Guill.\& Perr.) Suess. } \\
\text { Ceiba pentandra L. (Gaertn.) } \\
\text { Ceratotheca sesamoides Endl. } \\
\text { Cissus populnea Guill. \& Perr. } \\
\text { Coelocaryon oxycarpum Stapf } \\
\text { Cola caricaefolia (G. Don) K. Schum. } \\
\text { Corchorus olitorius L. } \\
\text { Curculigo pilosa (Schumach. \& Thonn.) Engl. } \\
\text { Detarium microcarpum Guill. \& Perr. } \\
\text { Dioscorea dumetorum (Kunth) Pax } \\
\text { Diospyros mespiliformis Hochst. ex A. DC. } \\
\text { Dracaena mannii Baker }\end{array}$ & $\begin{array}{l}\text { Malvaceae } \\
\text { Passifloraceae } \\
\text { Zingiberaceae } \\
\text { Zingiberaceae } \\
\text { Fabaceae } \\
\text { Araceae } \\
\text { Annonaceae } \\
\text { Phyllanthaceae } \\
\text { Balanitaceae } \\
\text { Sapindaceae } \\
\text { Malvaceae } \\
\text { Arecaceae } \\
\text { Euphorbiaceae } \\
\text { Meliaceae } \\
\text { Caricaceae } \\
\text { Vitaceae } \\
\text { Malvaceae } \\
\text { Pedaliaceae } \\
\text { Vitaceae } \\
\text { Myristicaceae } \\
\text { Malvaceae } \\
\text { Malvaceae } \\
\text { Hypoxidaceae } \\
\text { Fabaceae } \\
\text { Dioscoreaceae } \\
\text { Ebenaceae } \\
\text { Asparagaceae }\end{array}$ & $\begin{array}{l}\text { Arbre } \\
\text { Liane } \\
\text { Herbacée } \\
\text { Herbacée } \\
\text { Arbre } \\
\text { Herbacée, } \\
\text { Géophyte } \\
\text { Arbuste } \\
\text { Arbuste } \\
\text { Arbuste } \\
\text { Arbre } \\
\text { Arbre } \\
\text { Arbre } \\
\text { Arbuste } \\
\text { Arbre } \\
\text { Arbuste } \\
\text { Herbacée } \\
\text { Arbre } \\
\text { Herbacée } \\
\text { Liane } \\
\text { Arbre } \\
\text { Arbre } \\
\text { Herbacée } \\
\text { Herbacée } \\
\text { Arbre } \\
\text { Liane } \\
\text { Arbre } \\
\text { Arbuste }\end{array}$ & $\begin{array}{l}\text { A SZ me } \\
\text { A GC L } \\
\text { A SZ Gr } \\
\text { A SZ Gr } \\
\text { A GC-SZ me } \\
\text { A SZ G } \\
\text { A SZ-na } \\
\text { A SZ mi } \\
\text { palT SZ mi } \\
\text { panT GC-SZ me } \\
\text { A SZ mi } \\
\text { A GC-SZ mg } \\
\text { A GC-SZ mi } \\
\text { AN GC-SZ mi } \\
\text { N I mi } \\
\text { A GC Lmi } \\
\text { panT GC-SZ mg } \\
\text { A SZ Ch } \\
\text { A GC-SZ Lmi } \\
\text { A GCW me } \\
\text { A GCW mi } \\
\text { panT GC-SZ na } \\
\text { AM SZ H } \\
\text { A SZ mi } \\
\text { A GC-SZ G } \\
\text { A GC-SZ mi } \\
\text { A GC me }\end{array}$ \\
\hline
\end{tabular}

Tableau 1 (suite)

\begin{tabular}{l|l|l|l}
\hline Espèces & Famille & Morphologie & $\begin{array}{l}\text { Chorologie et type } \\
\text { biologique }\end{array}$ \\
\hline Elaeis guineensis Jacq. & Arecaceae & Arbre & A GC me \\
Ficus sur Forssk. & Moraceae & Arbre & A GC-SZ mi \\
Ficus vallis-choudae Delile & Moraceae & Arbre & A SZ mi \\
Flacourtia indica (Burn.f.) Merr. & Salicaceae & Arbuste & A SZ mi \\
Flueggea virosa (Roxb. ex Wild.) Voigt & Phyllanthaceae & Arbuste & palT GC-SZ na \\
Gardenia ternifolia Schumach. \& Thonn. & Rubiaceae & Arbuste & A SZ na \\
Grewia carpinifolia Juss. & Malvaceae & Arbuste & A GC mi \\
Hexalobus monopetalus (A.Rich) Engl. \& Diels & Annonaceae & Arbuste & A SZ mi \\
Hymenocardia acida Tul. & Hymenocardiaceae & Arbuste & A GC-SZ mi \\
Ipomoea cairica (L.) Sweet & Convolvulaceae & Liane & panT GC-SZ Lmi \\
\hline
\end{tabular}




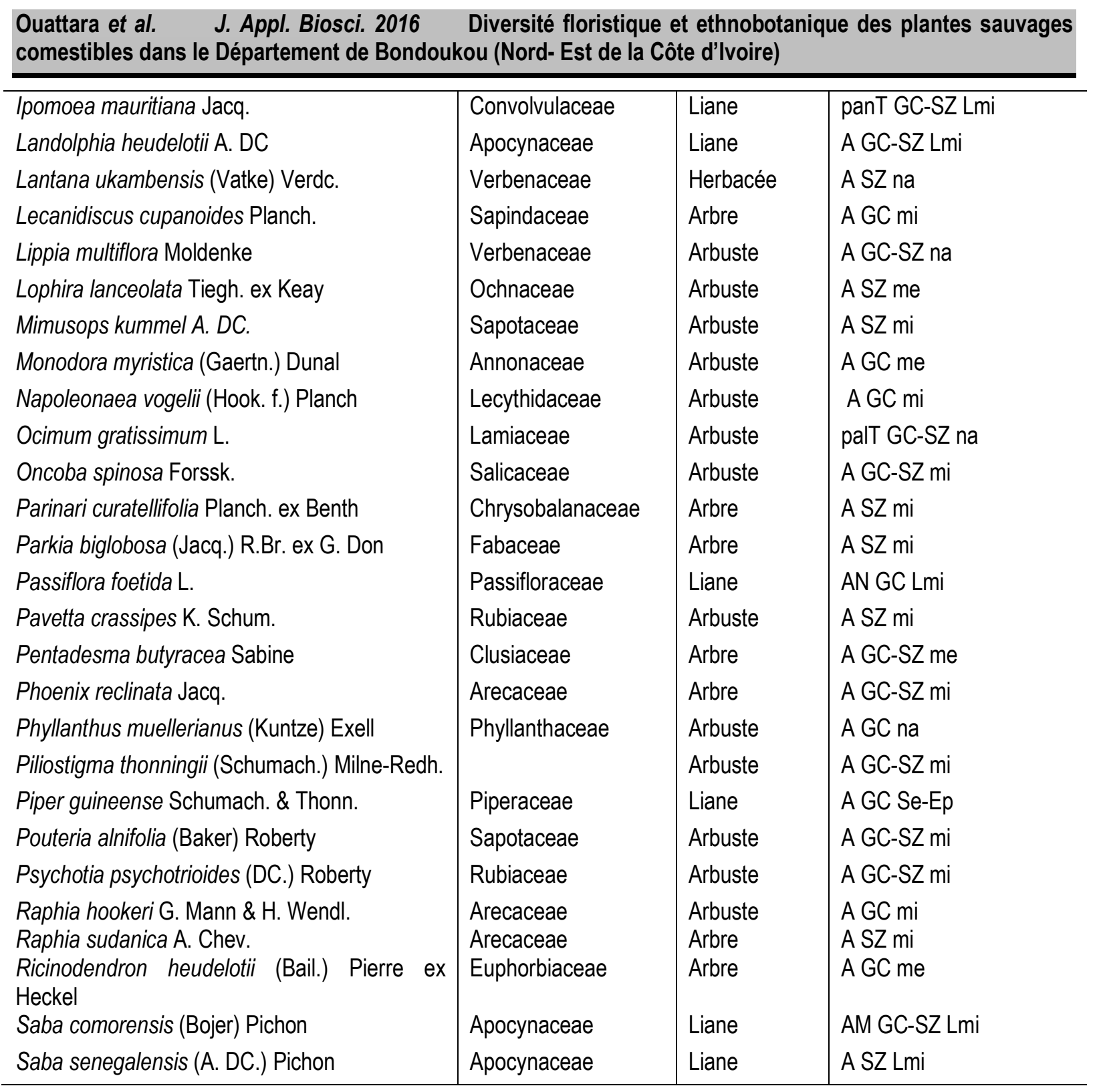

Tableau 1 (suite et fin)

\begin{tabular}{l|l|l|l}
\hline Espèces & Famille & Morphologie & $\begin{array}{l}\text { Chorologie et } \\
\text { type biologique }\end{array}$ \\
\hline Sarcocephalus latifolius (Smith) Bruce & Rubiaceae & Arbuste sarmenteux & A GC-SZ mi \\
Solanum torvum Sw. & Solanaceae & Arbuste & panT GC na \\
Spondias mombin L. & Anacardiaceae & Arbre & panT GC-SZ mi \\
Sterculia setigera Delile & Malvaceae & Arbre & A SZ mi \\
Strychnos spinosa Lam & Loganiaceae & Arbuste & AM SZ mi \\
Stylochiton hypogaeus Lepr. & Araceae & Herbacée & A SZ G \\
Talinum triangulare (Jacq.) Willd. & Talinaceae & Herbacée & AN GC na \\
Tamarindus indica L. & Fabaceae & Arbre & PanT GC-SZ mi \\
Uapaca togoensis Pax & Phyllanthaceae & Arbuste & A GC-SZ me \\
Uvaria chamae P. Beauv. & Annonaceae & Liane & A GC-SZ Lmi \\
\hline
\end{tabular}




\section{Ouattara et al. J. Appl. Biosci. $2016 \quad$ Diversité floristique et ethnobotanique des plantes sauvages comestibles dans le Département de Bondoukou (Nord- Est de la Côte d'Ivoire)}

\begin{tabular}{l|l|l|l}
\hline Vitex doniana Sweet & Lamiaceae & Arbre & Aco GC-SZ mi \\
Vitex madiensis Oliv. & Lamiaceae & Arbuste & A SZ mi \\
Vittelaria paradoxa C. F. Gaertn. & Sapotaceae & Arbre & A SZ mi \\
Xylopia aethiopica (Dunal) A. Rich & Annonaceae & Arbuste & A GC-SZ-me \\
Ximenia americana L. & Olacaceae & Arbuste & panT GC-SZ mi \\
Zanthoxylum zanthoxyloides (Lam.) Zepern. \& & Rutaceae & Arbuste & A GC-SZ mi \\
Timler & Rhamnaceae & Arbuste & palT SZ mi \\
Ziziphus mauritiana Lam. & Ari
\end{tabular}

me=mésophanérophyte=Arbre de 8 à $30 \mathrm{~m} ; \mathbf{m g}=$ mégaphanérophyte= Arbre de plus de $30 \mathrm{~m}$ de hauteur; na= nanophanérophyte=arbrisseau de 0,25 à $2 \mathrm{~m}$ de hauteur; $\mathrm{mi}=$ microphanérophyte=Arbuste de 2 à $8 \mathrm{~m}$ de hauteur; $\mathrm{L}=\mathrm{Liane}$; $\mathrm{Ch}=$ chaméphyte; $\mathbf{G}=$ Géophyte; $\mathbf{G r}=$ Géophyte rhizomateux; $\mathbf{H}=$ hémicryptophyte; Se-Ep= semi-épiphyte; $\mathbf{A}=$ taxon africain; $A c 0=t a x o n$ commun à l'Afrique et à l'Archipel des Comores; $\mathbf{A M = t a x o n}$ commun à l'Afrique et à Madagascar; $\mathbf{A N =}$ afronéotropical; $\mathbf{G C}=$ taxon de la région guinéo-congolaise (forêt dense humide); $\mathbf{G C W}=$ taxon endémique du bloc forestier à l'Ouest du Togo, comprenant le Ghana, la Côte d'Ivoire, le Libéria, la Sierra Léone, la Guinée, la Guinée Bissau, la Gambie et le Sénégal; I= introduit; N=néotropical; PalT= taxon paléotropical; commun à l'ancien monde (Afrique, Asie, Australie; lles du Pacifique); PanT= taxon pantropical; commun à tous les pays tropicaux du monde; $\mathbf{S Z}=$ taxon de la région soudanozambézienne (savanes, forêts claires). Base nomenclaturale et répartition géographique adaptées de Aké Assi (2001) et Chatelain et al. (2011).

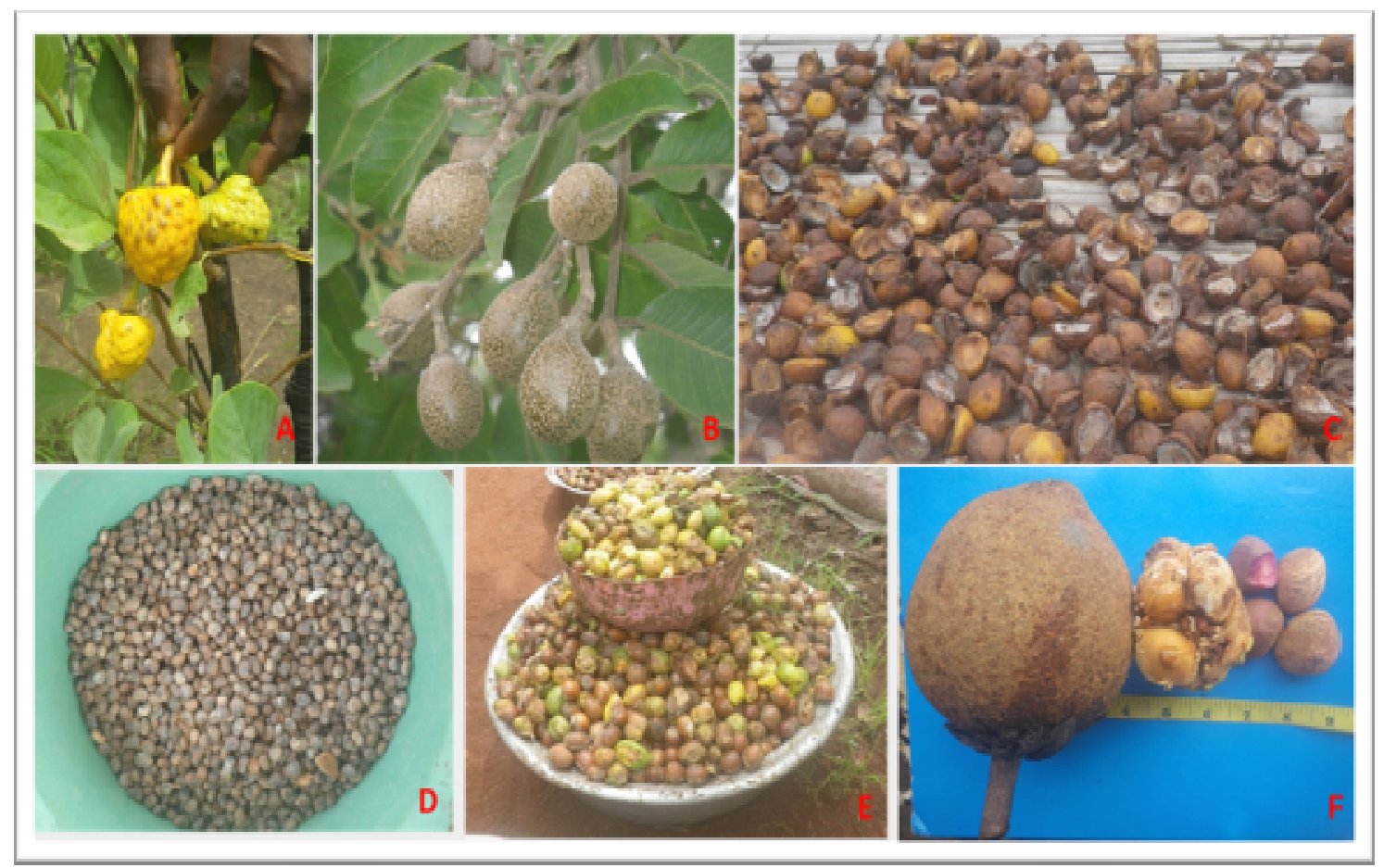

Figure 4 : Fruits et graines utilisés dans le Département de Bondoukou. A. Annona senegalensis; B. Parinari curatellifolia; C. Fruits de Coelocaryon oxycarpum en séchage; D. Graines de Adansonia digitata, E. Fruits de Vitellaria paradoxa ; F. Fruit et graines de Pentadesma butyracea 


\section{Ouattara et al. J. Appl. Biosci. 2016 Diversité floristique et ethnobotanique des plantes sauvages comestibles dans le Département de Bondoukou (Nord- Est de la Côte d'Ivoire)}

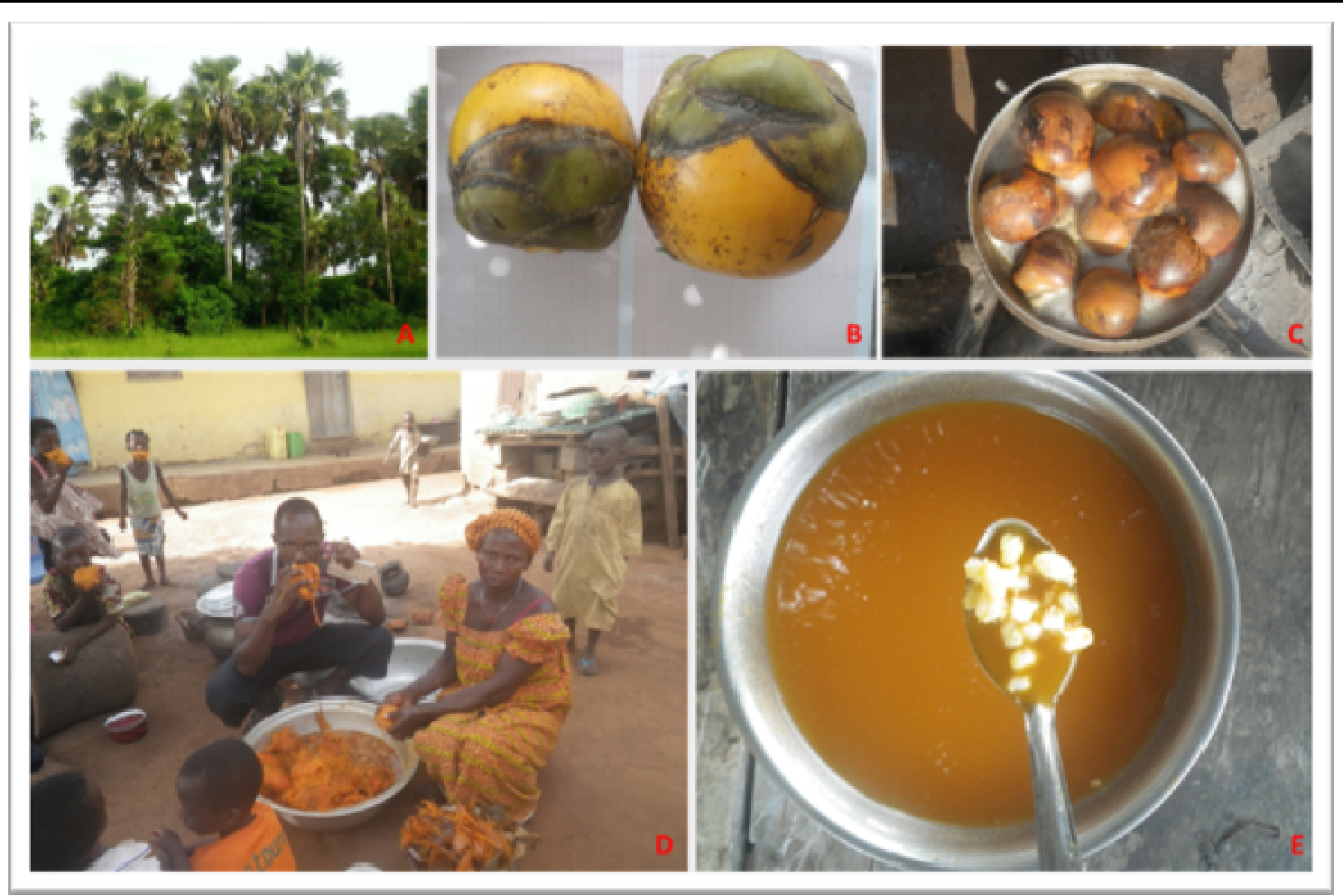

Figure 5 : Biologie et utilisation du palmier rônier (Borassus aethiopum). A. Habitus ; B. Fruits ; C. Fruits en cuisson ; $D$. Extraction du jus des fruits cuits. Jus mélangé au maïs cuit, prêt pour la consommation

\section{DISCUSSION}

Dans leur étude sur les plantes spontanées alimentaires de la région du Fromager à l'Ouest de la Côte d'Ivoire, Kouamé et al. (2008) ont inventorié 72 espèces appartenant à 42 familles. En comparant cette liste floristique à la nôtre, nous remarquons que seulement 13 espèces sont communes : Blighia sapida, Cayratia gracilis, Ceiba pentandra, Corchorus olitorius, Elaeis guineensis, Ocimum gratissimum, Piper guineense, Psychotria psychotrioides, Raphia hookeri, Ricinodendron heudelotii, Solanum torvum, Spondias mombin et Xylopia aethiopica. Parmi les espèces répertoriées par Kouamé et al. (2008), 59 ne figurent pas sur liste de la présente étude. De même, 68 espèces recensées au cours de cette étude ne sont pas citées par ces auteurs. Cette différence pourrait être liée à celle de la flore générale, vu que la région du Fromager est une zone de forêt dense humide semi décidue alors que notre étude a lieu dans une zone de transition savanes guinéennes-savanes subsoudanaises. Les travaux d'Ambé (2001), dans la région de Séguéla, au Nord-Ouest de la Côte d'Ivoire, ont porté exclusivement sur les fruitiers sauvages comestibles de cette région qui, du point de vu phytogéographique, serait semblable à notre zone d'étude. Ce chercheur a obtenu 75 espèces fruitières appartenant à 35 familles. Le terme "espèce fruitière» désigne ici les espèces dont les fruits et/ ou les graines sont consommés directement ou après transformation. Ainsi, dans notre travail, 56 espèces fruitières ont été recensées. Parmi les 72 espèces inventoriées par Ambé (2001), 38 ont été citées par nos informateurs tandis qu'ils n'ont pas fait cas de 37 autres espèces. En outre, 15 espèces fruitières recensées dans le cadre de notre travail ne se retrouvent pas chez ce chercheur. Gauthier-Béguin (1992) a étudié les plantes sauvages comestibles dans la région du V-Baoulé et a recensé 48 espèces. Parmi elles, 26 espèces dont 18 fruitières, ont été recensées comme plantes sauvages comestibles à Bondoukou tandis que les 22 autres n'ont pas été citées par nos informateurs. Certaines espèces de toutes ces études ont été bel et bien observées par nous dans notre site d'étude, mais n'ont pas été citées parmi les plantes comestibles par nos informateurs. Nous avons également recensés 24 espèces qui n'ont été citées dans aucune des trois études précédentes évoquée ici (Gauthier-Béguin, 1992 ; Ambé, 2001 ; Kouamé et al., 2008 ). II s'agit de Adenia cissampeloides, Aframomum melegueta, 
Anchomanes welwetchii, Afzelia africana, Balanites aegyptiaca, Coelocaryon oxycarpum, Dioscorea dumetorum, Dracaena mannii, Flacourtia vogeli, Flueggea virosa, Ipomoea cairica, Ipomoea mauritiana, Lantana ukambensis, Mimusops kummel, Monodora myristica, Oncoba spinosa, Pavetta crassipes, Phyllanthus muellerianus, Piliostigma thonningii, Pouteria alnifolia, Raphia sudanica, Sterculia setigera, Stylochiton hypogaeus et Zanthoxylum zanthoxyloides. Par ailleurs, parmi ces espèces, si l'usage alimentaire de 23 a été signalé dans la littérature, ce n'est pas le cas de Coelocaryon oxycarpum. En effet, son usage alimentaire n'est pas rapporté dans la littérature pour aucune autre région d'Afrique de l'Ouest et même de Côte d'Ivoire. Burkill (1985) indique l'utilisation de sa sève en médecine traditionnelle, comme un laxatif, mais ne fait aucune référence à l'usage alimentaire de ses fruits. Selon les utilisatrices, la consommation de la sauce faite avec ses fruits permet un rejet total des caillots de sang après l'accouchement et une cicatrisation rapide des blessures. Aucune étude phytochimique évaluant les composés jouant ces rôles n'a été réalisée au niveau de cette espèce. Toutes ces différences montrent que les habitudes, les savoirs et les usages des plantes diffèrent d'une région à l'autre, d'un peuple à l'autre et ce, parfois dans un même espace ethno-floristique.

Taxonomie locale: Au niveau des noms vernaculaires, plusieurs noms existent pour certaines espèces alors qu'un même nom peut désigner plusieurs espèces. Ambé (2001) a également fait ce constat dans la région de Séguéla chez les Malinké, au Nord-Ouest de la Côte d'lvoire. Les différences d'appellation existent parfois entre villages d'une même ethnie. Par exemple dans le village de Kiendi Walogo, Balanites aegyptiaca est connu sous le nom de Toulôbô-N'kpo qui signifie " épine d'éléphant » alors qu'à Sorobango (situé à $5 \mathrm{Km}$ ) la plante est appelée Toulôbô-togossogo signifiant "piment d'éléphant ". Chez les Nafana de San-Poro la plante est désignée par le terme sole-bôlô qui signifie aussi "épine d'éléphant». Beaucoup de noms de plantes ont une signification à l'instar de celles de Balanites aegyptiaca. Ainsi Afrabôgbadou (Passiflora foetida) signifie " banane douce de chat », Lomgbéio (Pavetta crassipes) signifie " oreille de lapin ", Kanguélépèssè (Parinari curatellifolia) signifie "cure dent de Kanguélé ". Kanguélé est un village de la région ou cette espèce pousse en abondance.

Des connaissances et pratiques tombées en désuétude: L'exploitation du vin à partir du palmier
Phoenix reclinata pendant les temps anciens a été signalée par les informateurs. Cependant cette pratique n'existe pratiquement plus dans la région. A Sorobango, les habitants disent que cette pratique a disparu avec l'avènement de l'Islam. En effet la population de ce village est majoritairement musulmane. L'usage alimentaire des huiles de Carapa procera et Lophira lanceolata est également de plus en plus rare. Les personnes interrogées expliquent cela par le fait que ces huiles ont un goût amer. Cependant, leur utilisation pour la fabrication de savons traditionnels locaux est bien courante dans la région. Pentadesma butyracea est moyennement connue et bien exploitée. Le niveau de connaissance moyen de l'espèce s'explique par le fait qu'elle est très localisée. En effet, on ne la trouve que dans des zones forestières autour du village de Laoudi Gan, ces dernières fortement menacées. Cependant, toutes les personnes ayant consommé le beurre (ou l'huile) de cette Clusiaceae affirment le préférer à celui de Vitellaria paradoxa (beurre de karité). Les personnes l'ayant reconnue hors du village de Laoudi Gan, essentiellement des femmes, affirment qu'avant il existait un commerce de cette huile dans la région et c'est pour cela qu'elles connaissent la plante. Aujourd'hui cette huile n'est quasiment plus vendue sur les marchés. Si l'on peut expliquer cela par la présence de l'huile industrielle (huile raffinée de palme), il est aussi probable que cela soit dû, au moins en partie, à la rareté de la plante, comme nous l'on affirmé certaines personnes interrogées à Laoudi Gan. Ces personnes exploitent donc les fruits de la plante pour leur propre consommation. En effet, la présence de l'huile industrielle n'a pas estompé la vente, ou la production pour les ménages, du beurre de Vitellaria paradoxa, espèce relativement bien représentée dans la région. Vraisemblablement de nombreuses populations de Pentadesma butyracea ont disparu de la région avec l'exploitation des zones forestières.

Possibilités de valorisation: La diversité des espèces, des usages et des organes utilisés offrent de nombreuses possibilités de transformation, de recherche et de valorisation pour la quasi-totalité des espèces. Les espèces à fruits charnus et juteux (par exemple Ximenia americana, Saba spp, Vitex spp,) peuvent faire l'objet de fabrication de jus naturels. Les possibilités de valorisation en cosmétique de Pentadesma butyracea, mériteraient d'être explorées. II en est de même pour Lophira lanceolata et Carapa procera, deux espèces déjà employées pour la fabrication de savons traditionnels. 


\section{CONCLUSION}

II ressort de cette étude que les plantes sauvages comestibles occupent encore une place prépondérante dans la vie quotidienne des populations dans le Département de Bondoukou. Cependant, les connaissances liées à l'utilisation de ces plantes se perdent au fil des années. On dénombre déjà 13 espèces peu connues et peu exploitées. Les usages sont également en mutation. Certains sont en abandon car de nouvelles alternatives s'offrent aux populations.

\section{REMERCIEMENTS}

Ce travail a été réalisé dans le cadre du doctorat $d u$ premier auteur, financé par le Programme d'Appui Stratégique à la Recherche Scientifique en Côte d'Ivoire (PASRES, projet $\mathrm{N}^{\circ}$ 142). Nos remerciements vont à l'endroit des Responsables et du personnel de ce programme. Nous sommes reconnaissants vis-à-vis des chefs de village et des populations des zones visitées, qui nous ont permis de travailler librement et qui ont toujours fait montre d'une bonne coopération. Merci aux Responsables du Centre Suisse de
L'inventaire des savoirs liés à la consommation de ces plantes est une étape fondamentale pour la lutte contre la perte des connaissances et de toutes les valeurs culturelles qui en sont liées. Les différences au niveau floristique et au niveau des usages, constatées entre notre zone d'étude et d'autres régions de la Côte d' Ivoire, mettent en exergue le fait qu'il est nécessaire de conduire un inventaire national des plantes sauvages comestibles.

Recherches Scientifiques en Côte d'Ivoire et à ceux de I'Unité de Formation et de Recherche des Sciences de la Nature (UFR SN) de l'Université Nangui Abrogoua, pour leur soutien logistique dans la réalisation de cette étude. Nous remercions notre guide et interprète, Ouattara Issouf, de Sorobango. Enfin, grand merci à Monsieur et Madame Mel Sess, Madame Diarrassouba Mariame (Mme cool) et Monsieur Ouattara Ali dit Ali technicien, pour leurs aides précieuses. 
Tableau 2. Niveaux de connaissance et d'exploitation, noms vernaculaires et organes consommés de 70 espèces.

\begin{tabular}{|c|c|c|c|}
\hline $\begin{array}{l}\text { Niveau de connaissance et de } \\
\text { consommation (exploitation) }\end{array}$ & Espèces & Noms vernaculaires (Koulango et/ou Nafana) & $\begin{array}{l}\text { Organes } \\
\text { consommés }\end{array}$ \\
\hline $\begin{array}{l}\text { Espèces bien connues et bien } \\
\text { exploitées }\end{array}$ & $\begin{array}{l}\text { Adansonia digitata }(90 / 85) \\
\text { Annona senegalensis }(100 / 100) \\
\text { Borassus aethiopum }(105 / 95) \\
\text { Carica papaya }(105 / 90) \\
\text { Corchorus olitorius }(90 / 70) \\
\text { Diospyros mespiliformis }(75 / 70) \\
\text { Elaeis guineensis }(105 / 102) \\
\text { Landolphia heudelotii }(90 / 55) \\
\text { Lippia multiflora }(100 / 75) \\
\text { Parkia biglobosa }(105 / 85) \\
\text { Pavetta crassipes }(85 / 60) \\
\text { Saba comorensis }(90 / 60) \\
\text { Saba senegalensis }(105 / 103) \\
\text { Strychnos spinosa }(60 / 35) \\
\\
\text { Tamarindus indica }(105 / 95) \\
\text { Vitex doniana }(103 / 98) \\
\text { Vitex madiensis Oliv. }(103 / 98) \\
\text { Vittelaria paradoxa }(105 / 104) \\
\text { Ximenia americana }(71 / 56)\end{array}$ & $\begin{array}{l}\text { Laakô (Koulango); Zin (Nafana) } \\
\text { Bémé (Koulango); Bemé (Nafana) } \\
\text { Sangnego (Koulango); Sangnô (Nafana) } \\
\text { Bêrêfêrê (Koulango); Borfêre (Nafana) } \\
\text { Fonongô, Kplala (Koulango); Ayôyô (Nafana) } \\
\text { Hignô (Koulango); Gnagno (Nafana) } \\
\text { Tchingo (Koulango); Sogowê, Abê (Nafana) } \\
\text { Fôdjô (Koulango); Fôdjô (Nafana) } \\
\text { Agakpayo (Koulango); Djangouro (Nafana) } \\
\text { Dôgô (Koulango); Ningnê (Nafana) } \\
\text { Youngbéio (Koulango); Céléwé (Nafana) } \\
\text { Payo, Hôra (Koulango); Solomiroué (Nafana) } \\
\text { Kouloukaga, Agnibrou (Koulango); Miroué (Nafana) } \\
\text { Siangbodjigbô, Koulikoukou (Koulango);Siakoumorô } \\
\text { (Nafana) } \\
\text { Tomi, Agaboyo (Koulango); Sanhan (Nafana) } \\
\text { Ankố (Koulango); Foumbé (Nafana) } \\
\text { Ankô (Koulango); Foumbé (Nafana) } \\
\text { Vakô (Koulango); Loho (Nafana) } \\
\text { Déyo, Dé (Koulango); Morô (Nafana) }\end{array}$ & $\begin{array}{l}\text { Fruit, graine } \\
\text { Fruit } \\
\text { Fruit } \\
\text { Fruit } \\
\text { Feuille } \\
\text { Fruit } \\
\text { Fruit } \\
\text { Fruit } \\
\text { Feuille } \\
\text { Fruit } \\
\text { Feuille } \\
\text { Fruit } \\
\text { Fruit } \\
\text { Fruit } \\
\\
\text { Fruit } \\
\text { Fruit } \\
\text { Fruit } \\
\text { Fruit et graine } \\
\text { Fruit }\end{array}$ \\
\hline
\end{tabular}

Tableau 2 (suite)

\begin{tabular}{l|l|l|l}
\hline $\begin{array}{l}\text { Niveau de connaissance et de } \\
\text { consommation (exploitation) }\end{array}$ & Espèces & Noms vernaculaires (Koulango et/ou Nafana) & $\begin{array}{l}\text { Organes } \\
\text { consommés }\end{array}$ \\
\hline & Blighia sapida (72/21) & Soungo (Koulango); Souho (Nafana) & Fruit (arille) \\
Cissus populnea (75/19) & Kpatcha-Kpatcha, Yolognô (Koulango); Fouro (Nafana) & Feuille \\
Espèces bien connues et & Detarium microcarpum (70/25) & Crocrowa (Koulango); Akoko (Nafana) & Gruit \\
moyennement exploitées & Monodora myristica (55/20) & Bassan (Koulango); Bassan (Nafana) & Fruit \\
& Parinari curatellifolia (60/15) & Kanglèpèssè (Koulango); Komoro (Nafana) & Graine \\
& Ricinodendron heudelotii (60/22) & Akpi, Api (Koulango); Akpi (Nafana) & Fruit \\
& Solanum torvum (71/30) & Dabré, Yabrê (Koulango); Bébréme (Nafana) & Fruit \\
& Uvaria chamae (56/14) & Sôgôfôdjô,, Gbaligbadou (Koulango); Talio (Nafana) & Fruit, graine \\
\hline
\end{tabular}




\begin{tabular}{|c|c|c|c|}
\hline $\begin{array}{l}\text { Espèces bien connues et peu } \\
\text { exploitées }\end{array}$ & $\begin{array}{l}\text { Bridelia ferruginea }(80 / 5) \\
\text { Ceiba pentandra }(90 / 5) \\
\text { Ficus sur }(55 / 5) \\
\text { Ficus vallis choudae }(55 / 5) \\
\text { Flacourtia vogeli }(60 / 15) \\
\text { Gardenia ternifolia }(80 / 18) \\
\text { Lophira lanceolata }(70 / 6) \\
\text { Phoenix reclinata }(68 / 10) \\
\text { Oncoba spinosa }(55 / 15) \\
\text { Raphia sudanica }(75 / 5) \\
\text { Stylochiton hypogaeus }(57 / 11) \\
\text { Sarcocephalus latifolius }(75 / 10)\end{array}$ & $\begin{array}{l}\text { Ridjo (Koulango); Lakorô (Nafana) } \\
\text { Tonhonko (Koulango); Célégué (Nafana) } \\
\text { Yiyoungo, Youngbinhin (Koulango); Sia (Nafana) } \\
\text { Yiyoungo, Youngbinhin (Koulango); Sia (Nafana) } \\
\text { Stadéyo (Koulango); Mouroumourou (Nafana) } \\
\text { Katôgôma (Koulango); Katôgôma (Nafana) } \\
\text { Bligo (Koulango); Gnanta (Nafana) } \\
\text { Sianli (Koulango); Sianli (Nafana) } \\
\text { Stadéyo (Koulango); Mouroumourou (Nafana) } \\
\text { Kpagalego (Koulango); Kpanah (Nafana) } \\
\text { Kakotégo (Koulango); Boubon-nonnié (Nafana) } \\
\text { Youssèbôdigô, Issienkô, Balomé (Koulango); Siwé (Nafana) }\end{array}$ & $\begin{array}{l}\text { Fruit } \\
\text { Feuille } \\
\text { Feuille } \\
\text { Feuille } \\
\text { Fruit } \\
\text { Fruit } \\
\text { Graine } \\
\text { Fruit } \\
\text { Fruit } \\
\text { Fruit } \\
\text { Feuille } \\
\text { Fruit }\end{array}$ \\
\hline
\end{tabular}

Tableau 2 (suite)

\begin{tabular}{|c|c|c|c|}
\hline $\begin{array}{l}\text { Niveau de connaissance et de } \\
\text { consommation (exploitation) }\end{array}$ & Espèces & Noms vernaculaires (Koulango et/ou Nafana) & $\begin{array}{l}\text { Organes } \\
\text { consommés }\end{array}$ \\
\hline $\begin{array}{l}\text { Espèces } \\
\text { moyennement connues et } \\
\text { bien exploitées }\end{array}$ & $\begin{array}{l}\text { Aframomum alboviolaceum }(\mathbf{5 0 / 4 0 )} \\
\text { Cayratia gracilis }(\mathbf{5 0 / 4 5 )} \\
\text { Dracaena mannii }(\mathbf{3 0} / 27) \\
\text { Ipomoea mauritiana }(\mathbf{4 3 / 2 1 )}\end{array}$ & $\begin{array}{l}\text { Agateyo (Koulango); Ladjin (Nafana) } \\
\text { Waga-Waga (Koulango); Waga-waga (Nafana) } \\
\text { Kouabrêbê (Koulango) } \\
\text { Gbalimouta (Koulango); Lome (Nafana) }\end{array}$ & $\begin{array}{l}\text { Fruit } \\
\text { Feuille } \\
\text { Feuille } \\
\text { Feuille }\end{array}$ \\
\hline $\begin{array}{l}\text { Espèces moyennement } \\
\text { connues et moyennement } \\
\text { exploitées }\end{array}$ & $\begin{array}{l}\text { Ceratotheca sesamoides }(\mathbf{4 3 / 1 2}) \\
\text { Talinum triangulare }(\mathbf{2 7 / 1 0 )}\end{array}$ & $\begin{array}{l}\text { Kouara-n'gbô (Koulango); Fouro (Nafana) } \\
\text { Tchingosaladi, Douko (Koulango); Boulé (Nafana) }\end{array}$ & $\begin{array}{l}\text { Feuille } \\
\text { Feuille }\end{array}$ \\
\hline $\begin{array}{l}\text { Espèces moyennement } \\
\text { connues et peu exploitées }\end{array}$ & $\begin{array}{l}\text { Afzelia africana }(45 / 8) \\
\text { Anchomanes welwetchii }(\mathbf{4 3} / 10) \\
\text { Carapa procera }(35 / 1) \\
\text { Hexalobus monopetalus }(\mathbf{4 0 / 5 )} \\
\text { Hymenocardia acida }(35 / 3) \\
\text { Lecanidiscus cupanoides }(30 / 5) \\
\text { Ocimum gratissimum }(\mathbf{4 0 / 4 )}\end{array}$ & $\begin{array}{l}\text { Sakpô (Koulango); Tchégué (Nafana) } \\
\text { Dêdê (Koulango); Dêhê (Nafana) } \\
\text { Kloupia (Koulango); Kloupia (Nafana) } \\
\text { Yêrêbôpayo (Koulango); Lamarato (Nafana) } \\
\text { Paradjo (Koulango); Toukpa (Nafana) } \\
\text { Kloklodjô (Koulango) } \\
\text { Kouloukassigo (Koulango); Tchasségbô (Nafana) }\end{array}$ & $\begin{array}{l}\text { Feuille } \\
\text { Feuille } \\
\text { Graine } \\
\text { Fruit } \\
\text { Feuille } \\
\text { Fruit } \\
\text { Feuille }\end{array}$ \\
\hline $\begin{array}{l}\text { Espèces peu connues et bien } \\
\text { exploitées }\end{array}$ & $\begin{array}{l}\text { Coelocaryon oxycarpum }(20 / 18) \\
\text { Pentadesma butyracea }(20 / 18)\end{array}$ & $\begin{array}{l}\text { Brima (Koulango); Brima (Nafana) } \\
\text { Tchélépé (Koulango); Tchélépé (Nafana) }\end{array}$ & $\begin{array}{l}\text { Fruit } \\
\text { Graine }\end{array}$ \\
\hline $\begin{array}{l}\text { Espèces peu connues et } \\
\text { moyennement exploitées }\end{array}$ & $\begin{array}{l}\text { Balanites aegyptiaca (20/5) } \\
\text { Piper guineense (20/7) }\end{array}$ & $\begin{array}{l}\text { Toulôbô-N'kpo, Toulôbô-togossogo (Koulango); } \\
\text { Sole-bolô (Nafana) } \\
\text { Kpessi (Koulango): Kpessi (Nafana) }\end{array}$ & $\begin{array}{l}\text { Fruit } \\
\text { Fruit }\end{array}$ \\
\hline
\end{tabular}

Tableau 2 (suite et fin) 


\begin{tabular}{l|l|l|l}
\hline $\begin{array}{l}\text { Niveau de connaissance et de } \\
\text { consommation (exploitation) }\end{array}$ & Espèces & Noms vernaculaires (Koulango et/ou Nafana) & $\begin{array}{l}\text { Organes } \\
\text { consommés }\end{array}$ \\
\hline & Antidesma venosum (25/3) & Kakapepe (Koulango) & Fruit \\
& Bdegré (Koulango) & Fleur (pétale) \\
& Curculigo pilosa (10/1) & Foanbôdô-n'gbô (Koulango); Louépi (Nafana) & Fruit \\
& Flueggea virosa (15/3) & Lékrégno (Koulango); Lifa (Nafana) & Fruit \\
& Lantana ukambensis (20/3) & Lobizôgô (Koulango); Bounssibi (Nafana) & Fruit \\
& Passiflora foetida (20/2) & Afrabôgbadou (Koulango); Djaramoissibiè (Nafana) & Fruit \\
Espèces peu connues et peu & Phyllanthus muellerianus (15/2) & Galangalanhandio (Koulango); Sogokènè (Nafana) & Fruit \\
exploitées & Psychotria psychotrioides (15/3) & Hidjigabôdêkô (Koulango); Guinda (Nafana) & Feuille \\
& Spondias mombin (10/1) & Tagalema (Koulango);Tagalema (Nafana) & Fruit \\
& Sterculia setigera (15/2) & Borogodidigo, Drougo, Wolidjo (Koulango); Kotôgô & Graine \\
& & (Nafana) & \\
& Gapaca togoensis (15/2) & Gnom-dôbi (Koulango) & Fruit \\
& Zanthoxylum zanthoxyloides (11/2) & Hangbô (Koulango); Kaga (Nafana) & Feuille \\
& Ziziphus mauritiana (10/2) & Dakoumana (Koulango); Wandabor (Nafana) & Fruit \\
\hline
\end{tabular}

Devant chaque espèce et entre parenthèse : le premier nombre (en gras) représente le nombre de personnes ayant reconnu la plante parmi 105 personnes interrogées, le second nombre (sous la barre) est le nombre de personnes ayant consommé l'espèce au moins une fois parmi les personnes ayant reconnu l'espèce 


\section{RÉFÉRENCES}

Aké Assi L, 2001. Flore de la Côte d'Ivoire: Catalogue Systématique, Biogéographie et écologie. II. Boissiera 57:1-396.

Ambé GA, 2001. Les fruits sauvages comestibles des savanes guinéennes de la Côte d'Ivoire : état de la connaissance par une population locale, les Malinké. Biotechnologie, Agronomie, Société \& Environnement 5 (1) : 43-58.

Ayessou NC, Gueye M, Dioh E, Konteye M, Cisse M, Dornier M, 2008. Composition nutritionnelle et apport énergétique du fruit de Maerua pseudopetalosa, aliment de soudure au Sénégal. Fruits 64 (3): 1-9.

APG III, 2009. An update of the Angiosperm Phylogeny Group classification for the orders and families of flowering plants: APG III, Botanical Journal of the Linnean Society 161:105-121.

Barima YSS, Barbier N, Bamba I, Traore D, Lejoly J, Bogaert J, 2009. Dynamique paysagère en milieu de transition forêt-savane. Bois et Forêts des Tropiques 299 (1) : 15-25.

Burkill HM, 1985. The useful plants of west tropical Africa: Families M-R. Vol 4. Royal Botanic Gardens

Chatelain C, Aké Assi L, Spichiger R, Gautier L, 2011. Cartes de distribution des plantes de Côte d'Ivoire. Boissiera 64:1-327.

Gautier-Beguin D, 1992. Plantes de cueillette alimentaires dans le sud du V-Baoulé en Côte d'Ivoire. Boissiera 46:1-341.

Guillaumet J-L et Adjanohoun E 1971. La végétation de la Côte d'Ivoire. In Avenard JM, Eldin E, Girard G, Sircoulon J, Touchebeuf P, Guillaumet J-L, Adjanohoun E, Perraud A (eds). Le milieu naturel de la Côte d'Ivoire. ORSTOM, Paris. P 157-266.

Herzog FM, 1992. Étude biochimique et nutritionnelle des plantes alimentaires sauvages dans le sud du V-Baoulé, Côte d'lvoire. Thèse de doctorat. EPFZ, Suisse, $122 p$

Herzog F, Farah Z, Amado R, 1994.Composition and consumption of gathered wild fruits in the $\mathrm{V}$ Baoulé, Côte d'lvoire. Ecology of Food and Nutrition 32:181-196.

Iranbakhsh A, Ebadi M, Zare Z, 2009. The contribution of indigenous fruit trees in sustaining rural livelihoods and conservation of natural resources. Journal of Horticulture and Forestry 11 (1): $1-6$

Lamien-Meda A, Lamien CE, Compaore MMY, Meda RNT, Kiendrebeogo M, Zeba B, Millogo JF,
Nacoulma OG, 2008. Polyphenol content and antioxidant activity of fourteen wild edible fruits from Burkina Faso. Molecules 13: 581-594.

Morel R, 2004. Le Climat et l'implantation des hommes le cas de la Côte d'lvoire. Annales de l'Association de Climatologie 1: 117-132.

Musinguzi E, Kikafunda JK, Kiremire BT, 2007. Promoting indigenous wild edible fruits to complement roots and tuber crops in alleviating vitamin A deficiencies in Uganda. Proceedings of the $13^{\text {th }}$ ISTRC Symposium, Arusha, Tanzania, PP. 763-769.

Kouamé N'M-T et Gnahoua GM, 2008. Arbres et lianes spontanés alimentaires du département de Gagnoa (centre-ouest de la Côte d'Ivoire). Bois et Forêts des Tropiques 298 (4) : 65-75.

Kouamé N'M-T, Gnahoua GM, Kouassi KE, Traoré D, 2008. Plantes alimentaires spontanées de la région du Fromager (Centre-Ouest de la Côte d'Ivoire): flore, habitats et organes consommés. Sciences \& Nature 5 (1) : 61-70.

Kouamé N'M-T, Gnahoua GM, Mangara A, 2014. Essais de germination de Ricinodendron heudeloti (Euphorbiaceae) dans la région du Fromager au centre-ouest de la Côte d'Ivoire. Journal of Applied Biosciences 56: 4133-4141.

Ouattara N', 2001. Situation des ressources génétiques forestières de la Côte d'lvoire (zone de savanes). Atelier sous-régional $\mathrm{FAO}$ IPGRI/CIRAF sur la conservation, la gestion, l'utilisation durable et la mise en valeur des ressources génétiques forestières de la zone sahélienne (Ouagadougou, 22-24 sept. 1998) (http://www.fao.org/forestryForestry.asp).

Rufo KC, Birnie A, Engnäs B, 2002. Edible wild plants of Tanzania. Regional Land Management Unit (RELMA). Technical note handbook No. 27. $780 \mathrm{p}$

Schreckenberg K, Awono A, Degrande A, Mbosso C, Ndoye O, Tchoundjeu Z, 2006. Domesticating indigenous fruit trees as a contribution to poverty reduction. Forests, Trees and Livelihoods 16: $35-51$

Thiombiano DNE, Lamien N, Dibong SD, Boussin IJ, 2010. Etats des peuplements des espèces ligneuses de soudure des communes rurales de Pobé-Mengo et de Nobéré (Burkina Faso). Journal of Animal \& Plant Sciences 9 (1): 1104-1116. 